\title{
Shareholder engagement e co-creation. Un'analisi su un campione di imprese quotate ${ }^{1}$
}

\author{
Salvatore Esposito De Falco - Nicola Cucari - Sergio Carbonara
}

\begin{abstract}
Obiettivi del paper: Il lavoro analizza e interpreta lo shareholder engagement quale strumento di co-creazione del valore e intende verificare se quest'ultimo possa favorire una maggiore circolazione delle informazioni all'interno dell'impresa, creando le premesse per un governo condiviso.

Metodologia: Dato il carattere esplorativo dello studio, ci si è avvalsi di un approccio fenomenologico. Nel lavoro si utilizza un campione relativo al $44 \%$ di società italiane a larga e media capitalizzazione. I dati utilizzati, relativi al triennio 2014/2016, riportano il grado di dissenso delle minoranze rispetto alle proposte dell'assemblea degli azionisti relative alle politiche di remunerazione.

Risultati: Il lavoro evidenzia che politiche continue di shareholder engagement riducono il grado di dissenso delle minoranze verso le decisioni assembleari, soprattutto quelle relative alle politiche di remunerazione.

Limiti della ricerca: Lo studio è testato su un campione relativo al $44 \%$ delle società italiane a larga e media capitalizzazione quotate. Sarebbe auspicabile, in futuro, allargare l'indagine ad un campione di imprese internazionali, operanti in contesti differenti.

Implicazioni pratiche: Il lavoro offre informazioni circa il funzionamento e lefficacia delle azioni e delle politiche di engagement fornendo utili indicazioni pratiche sull'efficacia dei proxy solicitors e su un nuovo ruolo dell'assemblea.

Originalità del paper: Negli studi sulla corporate governance, lapproccio value co-creation non ha trovato uno specifico inquadramento epistemologico. In questo lavoro, invece, si è cercato di reinterpretare lo shareholder engagement come strumento di co-creation.
\end{abstract}

Parole chiave: shareholder engagement; value co-creation; corporate governance; dissenso societario

Purpose of the paper: The paper analyzes and interprets the shareholder engagement as a means of co-creation of value and aims to verify whether it could encourage a greater flow of information within the firm, creating the foundations for a shared government.

Methodology: This study has an exploratory approach; the scholars used a phenomenological approach to test a sample of $44 \%$ of Italian companies to large and mid-cap. The data used refer to the years 2014/2016 and report the degree of dissent

1 Pur essendo il lavoro frutto di unelaborazione congiunta degli autori, tuttavia il par. 3 è attribuibile a Carbonara; i par. 2 e 4 sono attribuibili a Cucari; i par. 1 e 5 sono attribuibili a Esposito De Falco. 
sinergie

italian journal of management

Vol. 36, N. 106, 2018

by minority shareholders with respect to the proposals in shareholder meetings and in particular with regard to remuneration policies.

Findings: Results show that the continuous shareholder engagement reduces the degree of dissent by minority towards business decisions, with regard to decisions relating to the remuneration policies.

Research limits: The paper used a sample of $44 \%$ of Italian companies to large and mid-caps listed. It would be desirable, in the future, expand the survey to a sample of international companies, operating in different contexts.

Practical implications: The paper provides information on the operation and effectiveness of the actions and policies of engagement by providing useful practical guidance on the effectiveness of proxy solicitors and on a "new role" of the Annual General Meeting.

Originality of the paper: In studies on corporate governance, the co-creation of value approach has not found a specific epistemological framework. In this work, however, we have tried to reinterpret the shareholder engagement as a co-creation tool.

Key words: shareholder engagement; value co-creation; corporate governance; dissent of minority shareholders'

\section{Introduzione}

Nell'impresa a capitale moderatamente concentrato le dinamiche di governo sono sempre state caratterizzate da una dialettica tra azionisti di maggioranza e minoranza che, il più delle volte, ha visto il prevalere degli interessi degli azionisti di comando su quelli comandati. Tale tendenza ha favorito l'insorgere di fenomeni d'acquisizione di benefici privati di controllo (self-dealing, dilution, reputation, ecc) a danno delle minoranze (Dyck e Zingales, 2004; Ehrhardt e Nowak, 2003; Esposito De Falco, 2014). In Italia i dati sulla concentrazione proprietaria degli ultimi anni mostrano una sostanziale stabilità della quota media detenuta dal principale azionista (47,1\% nel 2015; 46,2 \% nel 2010. Cfr. Consob, 2016); la qual cosa, inevitabilmente, induce ad immaginare la prevalenza degli interessi degli azionisti di maggioranza su quelli di minoranza (Doidge et al., 2009).

Negli ultimi anni, tuttavia, in Italia ed in altri contesti latini sembra stia emergendo una forte partecipazione degli investitori istituzionali stranieri alle dinamiche di governo dell'impresa, dovuta prevalentemente ad una maggiore predisposizione degli stessi ad azioni di voice ${ }^{2}$ ed a nuove normative (Shareholders' Rights Directive - 2007/36/EC) ${ }^{3}$.

2 Alcuni dati mostrano una maggiore partecipazione degli investitori istituzionali (in particolare esteri) alla nomina degli organi sociali delle società quotate italiane, alle decisioni assembleari, al controllo delle attività e delle scelte dell'azionista di maggioranza. Nelle assemblee delle società italiane incluse negli indici Ftse Mib e Mid Cap, infatti, è cresciuta la presenza degli investitori istituzionali esteri, passata dal 13\% nel 2012 al 21\% nel 2016; laddove quella degli investitori istituzionali italiani è rimasta sostanzialmente stabile, attestandosi nel 2015 all' $1,1 \%$ del capitale in assemblea (Consob, 2016, p. 86).

3 Nel Marzo 2017, la disciplina europea sui diritti degli azionisti ha subito ulteriori modifiche, focalizzandosi maggiormente sullorizzonte temporale di 
I dati relativi ad una maggiore diffusione dell'attivismo societario dimostrano come il quorum medio di voto nelle società del FTSE MIB (escluse le società cooperative e le società costituite al di fuori dell'Italia) è passato dal 52,3\%, nel 2010 (Georgeson, 2011), al 66,4\%, nel 2016 (dati indipendenti è salito dal $20,5 \%$ al $43,7 \%$ delle azioni rappresentate in assemblea. Inoltre, durante la crisi finanziaria del 2007-2009, gli azionisti strategici (tra cui lo Stato italiano) non sono stati in grado di mantenere le loro posizioni di controllo e sono stati costretti a diluire la loro quota media dal 42,7\% del 2010 al 39,2\% del capitale delle aziende FTSE MIB (escluse le banche cooperative).

Lattivismo e la partecipazione alle assemblee degli azionisti istituzionali tende, quindi, a modificare il grado di salience degli altri stakeholder aziendali, i quali mostrano un atteggiamento più attivo nei confronti delle dinamiche aziendali, anche partecipando al processo di creazione del valore aziendale (Mitchell et al., 1997). Quest'ultimo aspetto va inteso come partecipazione di tutti gli shareholder alle decisioni aziendali.

In quest'ottica l'emergere dell'importanza degli investitori istituzionali nella co-creazione del valore aziendale e, nella fattispecie, nella dialettica di governo, implica un ripensamento del rapporto tra proprietàmanagement, azionisti e società emittenti, impresa e stakeholders, favorito da sempre più diffuse politiche di engagement volte a stimolare ed allargare la partecipazione degli azionisti alle decisioni aziendali.

In particolare, mentre in una precedente fase storica l'engagement si limitava ad eventi in prossimità dell'assemblea annuale e/o ad situazioni di crisi (Mehta, 2013), oggi, in seguito alle mutate condizioni ed ai processi di globalizzazione in atto, si registra un rafforzamento dell'engagement, che tende a favorire in modo continuativo (continuative shareholder engagement) la partecipazione di tutti gli shareholder alla vita societaria.

Un esempio è quello di BlackRock ${ }^{4}$ che, in più occasioni, ha inviato messaggi alle società partecipate, incoraggiandole ad adottare un atteggiamento di lungo periodo nelle scelte di investimento e di governo aziendale. La stessa Commissione Europea, recentemente, si è interrogata sul fenomeno dell'engagement degli azionisti a lungo termine ed in merito

lungo periodo da parte di tutti gli azionisti. Uno dei motivi di tale revisione è specificamente la crisi finanziaria, in quanto le carenze della corporate governance hanno contribuito alla stessa. In particolare, ci si riferisce: al mancato impegno e controllo da parte degli azionisti nei confronti del processo decisionale delle società; alla retribuzione eccessiva e non giustificata da livelli di performance, che ha contribuito a creare un senso di sfiducia tra azionisti e società in generale; alle costose procedure per l'esercizio dei diritti degli azionisti. Per un approfondimento si rimanda al sito: http://europa.eu/rapid/ press-release_MEMO-17-592_en. htm

4 Larry Fink, l’amministratore delegato di BlackRock, il più grande investitore del mondo con $\$ 4.6$ miliardi di dollari gestiti, ha inviato una lettera annuale ai CEO delle aziende S\&P500 e le maggiori aziende europee, focalizzata sui temi della corporate governance. È possibile leggere la lettera inviata nel Febbraio 2016 al seguente link: https://www.blackrock.com/corporate/en-br/literature/ press-release/2016-larry-fink-ceo-letter.pdf
Salvatore Esposito De Falco Nicola Cucari

Sergio Carbonara

Shareholder engagement e

co-creation.
Unanalisi su un campione di imprese quotate 
sinergie Vol. 36, N. 106, 2018

all'esplicitarsi della corporate governance all'interno di società quotate ${ }^{5}$ (Collovà e Prestidge, 2015).

Partendo da tali considerazioni, nel presente lavoro si cercherà di analizzare e interpretare lo shareholder engagement quale strumento di co-creazione del valore, attraverso un'analisi degli effetti che lo stesso è in grado di produrre sulle dinamiche di governo. Più in generale, l'obiettivo è quello di arricchire le conoscenze in tema di shareholder engagement nell'ottica della co-creation. Da ciò la domanda di ricerca, in cui si chiede se lo shareholder engagement possa favorire un clima aziendale in grado di indurre una più diffusa partecipazione al governo societario ed una maggiore convergenza degli interessi di tutti gli azionisti.

In questo senso la co-creation va contestualizzata con riferimento ai seguenti aspetti:

1. Co-creation intesa come maggiore dialogo degli shareholder in merito alle decisioni assembleari: in un contesto di sempre minore concentrazione dell'azionariato $^{6}$ gli emittenti devono necessariamente riformulare le procedure di shareholder engagement, allargando la platea da un numero ristretto di azionisti di riferimento all'universo variegato degli investitori istituzionali; ma anche rivedere le pratiche stesse di governo aziendale, favorendo l'introduzione di best practice internazionali che tengano conto del maggiore potere di oversight e voice della generalità degli azionisti. In questottica lo shareholder engagement, se adottato come misura continuativa, riduce la percentuale di dissenso delle minoranze verso le decisioni proposte dal Board, favorendo l'instaurarsi di un clima condiviso più ampio e diffuso;

2. Co-creation intesa come maggiore convergenza degli interessi in gioco tra tutti gli azionisti: un dialogo continuo e trasparente con la generalità degli azionisti di lungo periodo, soprattutto se esteri, favorisce una maggiore convergenza degli interessi tra gli azionisti ed una più diretta comprensione delle specificità dell'emittente, del mercato e del settore in cui si opera, riducendo anche i rischi derivanti da una standardizzazione acritica delle valutazioni sulla corporate governance, che possono portare ad un'eccessiva devianza dai modelli prevalenti nel mercato (Acharya e Volpin, 2010; Aguilera et al., 2016). Ne è un esempio l'impatto che unazione continuata di shareholder engagement ha sulla riduzione del livello di dissenso nei casi di politiche di remunerazione proposte dal Board. Una maggiore adesione delle minoranze verso le

5 Gli autori identificano sei processi chiave "regarding the longevity of shareholder engagement and the quality of corporate governance within European listed companies. These are: 1) Insufficient shareholder engagement; 2) Insufficient link between pay and performance of directors; 3) Lack of shareholder oversight on related party transactions; 4) Doubts on the reliability of the advice of proxy advisors; 5) Difficult and costly exercise of rights flowing from shares; 6) Insufficient quality of corporate governance information" (Collovà e Prestidge, 2015).

http://www.europarl.europa.eu/RegData/etudes/BRIE/2015/528817/EPRS_ BRI(2015)528817_EN.pdf)

6 La minore concentrazione dell'azionariato è causata principalmente dalla riduzione, più o meno volontaria, delle quote degli azionisti di riferimento, e dal conseguente maggior peso degli investitori istituzionali, soprattutto con stile di investimento passivo e di lungo termine, nelle assemblee italiane. 
politiche di remunerazione proposte dal CdA, infatti, è frutto di una convergenza di interessi tra azionisti di maggioranza e minoranza.

Si rafforza, quindi, lidea di una corporate governance che tenga conto dell'effettivo ruolo e peso assunto dai diversi interlocutori dell'impresa, e non solo del capitale di comando, come sine qua non per determinare condizioni favorevoli alla co-creazione di valore.

In questo lavoro si intende dimostrare, infatti, che l'engagement riduce in modo significativo il grado di dissenso in assemblea, soprattutto se viene esercitato in modo continuo e non solo a ridosso delle assemblee stesse. In questo senso il lavoro è originale perché, nel contesto europeo, sono rare le ricerche empiriche focalizzate sui vantaggi dello shareholder engagement (Renneboog e Szilagyi, 2013).

Ciò premesso, il lavoro è così strutturato: dopo questa breve introduzione, nel secondo paragrafo $(\$ 2)$ si riporta il theoretical background dal quale si traggono le ipotesi di ricerca; successivamente si presenta la metodologia per l'indagine empirica $(\$ 3)$ e si analizzano i risultati $(\$ 4)$; infine, il lavoro si chiude con alcune considerazioni riguardanti il ruolo assunto dagli azionisti di minoranza nella prospettiva dello shareholder engagement e della co-creazione di valore (\$5).

\section{Theoretical background e ipotesi di ricerca}

Il tema del valore è da sempre presente negli studi economicofinanziari e di economia di impresa. La creazione di valore è ampiamente riconosciuta come l'obiettivo che il soggetto economico deve perseguire per assicurare all'impresa sopravvivenza e sviluppo; infatti, se l'attività di governo è tesa a creare valore nel tempo, la probabilità di sopravvivenza dell'impresa viene progressivamente ad innalzarsi (Golinelli, 2000).

Lidea di "valore condiviso" (shared value), tuttavia, si sviluppa, nellambito degli studi economico-aziendali, con i contributi di Porter e Kramer (2006), che favoriscono, di fatto, la nascita di una "nuova concezione del capitalismo", definita come "policies and operating practices that enhance the competitiveness of a company while simultaneously advancing the economic and social conditions in the communities in which it operates" (Porter e Kramer 2011, p. 66).

In letteratura questa nozione di valore condiviso ha generato un lungo dibattito (Ranjan e Read, 2016); secondo alcuni, tale concetto si sovrappone ad altri già noti, quali la responsabilità sociale delle imprese (CSR); per altri, invece, il valore condiviso è più una buzzword che un concetto teorico (Dembek et al., 2016). Korhonen (2013) reinterpreta il valore condiviso nellottica dei bisogni, in quanto "needs are nested in human systems where value is co-created by different parties at the individual, organizational, and societal levels. Altri autori, infine, sottolineano la novità del concetto, osservando che riconduce ad un cambiamento sostanziale nel comportamento delle imprese (Crane et al. 2014; Porter e Kramer, 2014).

D’altra parte, la "value co-creation", nella letteratura di marketing, è diventato un approccio fondamentale per il raggiungimento di una maggiore customer experience in unottica di lungo periodo (Prahalad e
Salvatore Esposito De Falco Nicola Cucari Sergio Carbonara Shareholder engagement

co-creation.
Unanalisi su un campione di imprese quotate 
sinergie Vol. 36, N. 106, 2018

Ramaswamy, 2004). Le imprese coinvolgono i propri clienti in misura sempre maggiore, al fine di comprenderne i bisogni (needs) e sviluppare, di conseguenza, offerte migliori e con un valore superiore (Röndell et al., 2016). La co-creazione, in effetti, è diventata un termine ampiamente usato per descrivere un cambiamento di pensiero delle imprese verso un processo più partecipativo (Ind e Coates, 2013).

Questa tendenza delle imprese si accompagna, in letteratura, ad uno sviluppo di approcci teorici quali la "Service Theory" - secondo la quale il valore emerge dalla cooperazione tra clienti e fornitori di servizi (Lovelock e Gummesson, 2004; Vargo e Lusch, 2004) - e il modello di "Value Constellation" (Normann e Ramirez, 1994, p. 54) - secondo il quale i clienti e i partner della supply chain "sono uniti nella co-creazione di valore".

Negli studi delleconomia di impresa, e in particolare di corporate governance, l'approccio value co-creation, pur non trovando uno specifico inquadramento epistemologico, trova le sue determinanti all'interno di specifici filoni teorici. Secondo la Stakeholder Theory, infatti, qualunque sia il fine ultimo dell'impresa, i manager devono tener conto dei legittimi interessi di quei gruppi e individui che possono influenzare le attività aziendali (Donaldson e Preston, 1995; Freeman, 1994).

Ciò è evidente anche nella Stewardship Theory, un approccio sociologico che esalta l'aspetto collaborativo, più che conflittuale, delle relazioni instaurate con gli stakeholder dell'impresa (Davis et al., 1997; Esposito De Falco, 2006).

In questa sede, tuttavia, preme sottolineare come il concetto di cocreazione del valore metta in discussione le dinamiche del rapporto manager/proprietà e "controlling shareholders"/ "minority shareholders"; di conseguenza l'insieme delle relazioni che l'impresa instaura con tutti gli shareholder. In tal senso si inserisce l'idea di una governance allargata, che tenga conto di tutti quei meccanismi, interni ed esterni, destinati ad orientare l'attività di impresa (Barile e Gatti, 2007; Esposito De Falco, 2006).

Parimenti, ciò che emerge dalle best practice internazionali in tema di corporate governance sembra indurre la creazione di un sistema di norme e regole che favorisca un maggior dialogo tra tutti gli azionisti, sia di maggioranza sia di minoranza, riducendo l'asimmetria informativa tra di essi. In questa direzione va interpretato il ruolo degli azionisti istituzionali e del sistema di voice, quali, ad esempio, il "say on pay" (Esposito De Falco, 2017). Anche la Commissione Europea ha sottolineato le carenze inerenti il coinvolgimento, nel lungo termine, di tutti gli azionisti delle società quotate (Collovà e Prestidge, 2015). Più specificatamente, agli azionisti non vengono fornite sufficienti informazioni e di conseguenza non sono in grado di impegnarsi in maniera efficace e costante.

In altre parole, dalle best practice scaturisce la necessità di imporre allorgano di governo la definizione di strumenti di governo e di gestione operativa capaci di coinvolgere tutti gli shareholder aziendali nelle scelte di governo, nonché di favorire la partecipazione ai processi decisionali di tutti gli stakeholders aziendali rilevanti.

Per un approfondimento sulla teoria principal-principal, si rimanda a Young et al., 2008. 
In tale direzione si collocano gli studi sullo shareholder activism. Quest'ultimo ricomprende tutte le azioni intraprese dagli investitori per influenzare la gestione aziendale e il Board (Chung e Talaulicar, 2010; Chung e Wynn, 2014). Goranova e Ryan (2014, p. 1232) lo definiscono come "actions taken by shareholders with the explicit intention of influencing corporations' policies and practices".

Nella letteratura sull'attivismo societario si individuano, pertanto, due filoni di studio: il financial activism ed il social activism (Judge et al., 2010; Goranova e Ryan, 2014).

La natura del primo filone è imprenditoriale (Klein, Zur, 2009) e riguarda tematiche relative allo shareholder value (executive pay, composizione del board, ecc); il secondo filone esplora gli effetti dell'attivismo in relazione ai diversi stakeholders: ad esempio, valuta l'impatto ambientale della società, o la corporate social performance (Goodman et al., 2014). Tuttavia, al di là della tipologia di attivismo, gran parte degli studiosi sottolineano come l'engagement è visto come strategia di voice da parte degli azionisti di minoranza (Hirschman, 1970).

In quest'ottica l'engagement può assumere molte forme, come ad esempio una maggiore partecipazione degli azionisti di minoranza alle decisioni proposte dal board; oppure un'intensificazione del dialogo "dietro le quinte" con il management, anche per il tramite dei lead indipendent directors, ecc. (Lydenberg, 2007).

Pertanto, per shareholder engagement si intendono le modalità con cui gli azionisti comunicano le loro opinioni, preoccupazioni, idee, bisogni al Consiglio di Amministrazione o all'organo di gestione e, viceversa, i modi in cui il Board ed il management comunicano le loro prospettive agli azionisti (Goldstein, 2014).

Diversi sono gli studi quantitativi e teorici sulle determinanti del dialogo, mentre risulterebbe un gap su come avviene l'effettivo coinvolgimento della società e sugli effetti dello shareholder engagement (Chuah, 2017; Strampelli, 2017; Ferraro e Beunza, 2014).

Da un lato, Hockerts e Moir (2004) sottolineano che la funzione delle relazioni con gli investitori è essenziale per raccogliere la percezione del mercato in merito alle tante decisioni aziendali (in primis le questioni di responsabilità sociale). Inoltre, gli autori rilevano che il dialogo è sempre più bidirezionale.

Dall'altro lato, lo shareholder engagement influenza il dibattito interno all'impresa, sensibilizzando il top management sulle implicazioni morali delle decisioni e facendo eco alle preoccupazioni di tutti gli stakeholders riguardo alcune decisioni aziendali.

Sulla base di tali considerazioni è possibile formulare la prima ipotesi del lavoro:

$\mathrm{H} 1$. Lo shareholder engagement riduce il grado di dissenso verso le decisioni assembleari.

Altri autori hanno dimostrato un effetto positivo tra diritti degli azionisti (diritto di voto e/o forme di engagement al Board) e performance aziendale (Mallin e Melis, 2012). A tal proposito, Rehbein et al. (2013) descrivono quattro modalità di risposta del Board allo shareholder
Salvatore Esposito De Falco Nicola Cucari Sergio Carbonara

Shareholder engagement e

Un'analisi su un campione di imprese quotate 
sinergie Vol. 36, N. 106, 2018

activism: cercare di omettere la risoluzione; lasciare che la risoluzione sia messa al voto assembleare; acconsentire alla richiesta o impegnarsi in un dialogo. Gli autori ipotizzano che le risposte agli "attivisti azionisti" dipendano dalle caratteristiche aziendali. Adottando la prospettiva della resource dependency theory, gli autori analizzano specifiche caratteristiche dell'impresa quali la dimensione, la corporate governance e la composizione del board. I risultati rilevano che i manager sono più propensi ad impegnarsi in un dialogo con gli azionisti quando l'impresa è più grande, vi è più sensibilità alle istanze degli stakeholder, il CEO è anche presidente, la società ha una percentuale relativamente bassa di investitori istituzionali.

Ne consegue che le caratteristiche del Board, o una determinata composizione di corporate governance, può facilitare la comunicazione tra board ed azionisti, promuovendo una maggiore consonanza tra le parti interessate, con benefici nel breve e nel lungo periodo (Esposito De Falco et al., 2016).

In alcuni contesti, tuttavia, lo shareholder activism è frenato da rigidità strutturali legate alle caratteristiche del sistema capitalistico di riferimento. Secondo Ivanova (2017) lo shareholder activism è molto context-specific, per cui fattori quali il sistema di corporate governance e la regolamentazione di un determinato paese impattano sulle dinamiche di coinvolgimento.

Othman e Borges (2015, p. 428) evidenziano l'importanza dell'impegno di essere soci di minoranza attivi rispetto alle scelte degli azionisti di controllo. Ai soci di minoranza attivi si riconosce un ruolo di "cane da guardia" (Ameer e Rahman, 2009), con effetti positivi su molteplici aspetti, quali, ad esempio, lexecutive compensation (Ertimur et al., 2010).

Ne deriva che la corporate governance è più efficiente ed efficace in un ambiente in cui tutti gli shareholder (in particolar modo large shareholder e azionisti istituzionali) e i manager sono incoraggiati a condividere un orizzonte di più lungo periodo (ad esempio aumentando la fedeltà degli azionisti e i meccanismi di voice - Letza et al., 2004). Ciò implica un ripensamento della governance e dei suoi meccanismi, che si manifesta attraverso cambiamenti nella frequenza, nella struttura e nel design dell'engagement (Conference Board Governance Center, 2016; Chapman and Cutler LPP, 2016): incontri più frequenti, e non solo a ridosso dell'assemblea annuale; tematiche strategiche e risk-oriented; differente interlocutore a seconda dello specifico topic di interesse. Queste modifiche suggeriscono un cambiamento in atto, nella relazione tra investitori e impresa, che necessita di maggiore attenzione e riflessione.

Alcuni studi dimostrano come le risposte delle imprese ai tentativi di influenza degli azionisti possono variare a seconda dell'approccio utilizzato da quest'ultimi per stimolare il cambiamento. Un approccio più collaborativo, ad esempio, influenza positivamente la corporate environmental performance (Lee e Lounsbury, 2011).

Da qui si ipotizza che:

$\mathrm{H}$ 2: Lo shareholder engagement, se utilizzato come politica attiva, piuttosto che episodica, può generare una maggiore convergenza di interessi tra tutti gli azionisti, riducendone il grado di dissenso. 
Al fine di testare le ipotesi formulate, si è scelto di studiare più casi piuttosto che tentare di catturare un effetto statistico valido (Gifford, 2012). Tale approccio, tipicamente fenomenologico (phenomenon based approach), può arricchire di nuove insight le teorie esistenti, o generarne di nuove (Von Krogh et al., 2012).

Nella letteratura sulla corporate governance, d'altra parte, gli studi che adottano un approccio qualitativo sono numericamente inferiori, rispetto a quelli che adottano un approccio quantitativo (McNulty et al., 2013), ma non meno importanti. Tali studi contribuiscono ad ottenere una più profonda comprensione delle relazioni tra i soggetti chiave ed i meccanismi messi in atto, evidenziando come gli attori e le istituzioni di governo operano realmente (Aguilera et al., 2015).

Tale metodologia ha consentito di descrivere le pratiche di engagement adottate da società italiane di grandi dimensioni a capitale diffuso. Quest'ultime presentano un azionariato caratterizzato da una forte presenza di investitori istituzionali, tra gli azionisti di minoranza. Tra questi è dato riscontrare la presenza di investitori di lungo termine, che adottano una gestione passiva degli investimenti, ovvero definiscono i titoli da acquistare e detenere in portafoglio sulla base di indici di mercato, o di modelli quantitativi interni ${ }^{8}$.

Gli azionisti con strategia passiva, sebbene in termini lessicali sembri esserci una contraddizione, sono quelli più attivi nel dialogo con gli emittenti e nel voto assembleare. Tali azionisti, infatti, sono tendenzialmente propensi a rinunciare al potere di exit, quando i risultati non sono soddisfacenti, adottando, invece, meccanismi di voice per tutelare i propri interessi nel lungo termine.

In questi casi gli investitori istituzionali sembrano manifestare una certa sensibilità alla presenza di elevati standard di corporate governance, orientati ad una maggiore tutela della creazione di valore sostenibile nel lungo periodo e di una conseguente riduzione, per quanto possibile, dei rischi derivanti da un cattivo governo societario?.

Ciò premesso, la nostra analisi parte dall'esperienza diretta dell'unico proxy advisor italiano, Frontis Governance, soffermandosi sulle attività di engagement effettuate nel triennio 2014-2016 da 42 società italiane quotate sul Mercato Telematico Azionario di Borsa Italiana. Nella definizione del periodo di riferimento si è tenuto conto di due fattori:

8 Di fatto, tali investitori non hanno alcuna discrezionalità nelle decisioni di investimento, ma sono vincolati a mantenere in portafoglio i titoli, fin tanto che questi siano inclusi nell'indice di mercato di riferimento o rispettino $\mathrm{i}$ criteri stabiliti dai modelli interni.

$9 \quad$ Secondo una ricerca pubblicata dalla società di analisi e valutazione dei fondi Morningstar (maggio 2016), i portafogli gestiti con strategia passiva rappresentano circa il $20 \%$ del totale del patrimonio gestito mondiale ( $40 \%$ negli Stati Uniti) ed hanno evidenziato una crescita del 230\% negli ultimi 10 anni. Secondo un'elaborazione di Frontis Governance di dati forniti da Georgeson, nel 2016 gli investitori passivi rappresentano mediamente circa il 15\% degli azionisti istituzionali delle società italiane a maggiore capitalizzazione 
sinergie Vol. 36, N. 106, 2018
- la relativa novità di tale tipologia di engagement nel mercato italiano, che ha iniziato a manifestarsi dopo l'introduzione del voto assembleare sulle politiche di remunerazione, nel 2012;

- la necessità di considerare un campione omogeneo, riducendo il più possibile le distorsioni derivanti dalle mutazioni negli assetti proprietari e nelle società componenti il campione; in particolare escludendo le società che hanno trasferito la sede all'estero, o che non sono più quotate in Italia.

Il campione preso in considerazione rappresenta il 44\% delle società italiane a larga e media capitalizzazione, escluse le società incorporate all'estero, di cui 31 emittenti incluse nel FTSE MIB alla data dell'ultima assemblea annuale del 2016 (86\% del listino) e 11 appartenenti all'indice FTSE Italia Mid Cap (18\%). Si è così cercato di ottenere un campione che rappresentasse tutte le practice utilizzate dagli emittenti italiani (dall'assenza totale di engagement al confronto continuo con gli investitori), senza attribuire un eccessivo peso alla categoria più rappresentata sul mercato (assenza di engagement).

In estrema sintesi, $\mathrm{i}$ casi analizzati rappresentano una varietà di approcci e stili di engagement che possono essere suddivisi in 3 categorie:

1. totale assenza di confronto con investitori e proxy advisors (21 emittenti);

2. attività di engagement focalizzate esclusivamente su un argomento trattato in una prossima assemblea (11 emittenti);

3. società che hanno intrapreso attività e/o adottato una politica di confronto continuo con gli investitori, finalizzate ad implementare alcune pratiche di governance (10 emittenti).

Al fine di verificare le ipotesi di studio, si è analizzato l'andamento, nel triennio 2014-2016, di due specifiche variabili:

1. il dissenso medio degli azionisti di minoranza sulle proposte presentate dal Board o da azionisti rilevanti in assemblee ordinarie (H1);

2. il dissenso medio delle minoranze sulle politiche di remunerazione (H2).

\section{Analisi e discussione}

In una prima fase dell'analisi si è preso in considerazione il dissenso medio degli azionisti di minoranza sulle proposte presentate dal Board, $\mathrm{o}$ da azionisti rilevanti in assemblee ordinarie. Per garantire il più alto grado di omogeneità nell'analisi, si è deciso di escludere le assemblee straordinarie e le proposte presentate da azionisti di minoranza in sede assembleare. Nel primo caso, infatti, il grado di dissenso dipende in maniera significativa da eventi contingenti, straordinari, che possono non essere direttamente collegabili ad una buona o cattiva governance societaria (ad esempio, fusioni ed acquisizioni o aumenti di capitale). Si è anche ritenuto opportuno non prendere in considerazione tutte quelle risoluzioni che, non previste nell'avviso di convocazione, sono state proposte nel corso della seduta assembleare da azionisti di minoranza. Se l'esclusione di tali proposte risiede proprio nell'obiettivo dell'analisi (verificare gli effetti 
sul consenso di pratiche di engagement tra proprietà e management, da un lato, ed azionisti istituzionali, dall'altro), le proposte presentate in sede assembleare non possono essere votate da tutti gli azionisti che partecipano all'assemblea in delega. In particolare, si tratta di richieste di autorizzazione ad intraprendere azioni di responsabilità nei confronti di esponenti aziendali, che sono generalmente presentate da azionisti privati e sono respinte con percentuali di dissenso prossime al 100\%.

Di seguito si riportano i dati relativi al dissenso medio, da parte delle minoranze, sulle proposte in discussione nelle assemblee ordinarie (cfr. Tav.1).

Tav. 1: Dissenso medio delle minoranze allassemblea annuale

\begin{tabular}{|l|c|c|c|}
\hline & Dissenso medio $\begin{array}{c}\text { minoranze su proposte Assemblea } \\
\text { Ordinaria }\end{array}$ \\
\hline & 2014 & 2015 & 2016 \\
\hline Media campione & $20.4 \%$ & $18.7 \%$ & $17.2 \%$ \\
\hline No engagement & $24.1 \%$ & $21.7 \%$ & $22.5 \%$ \\
\hline Solo in prossimità di Assemblee & $20.3 \%$ & $19.2 \%$ & $17.2 \%$ \\
\hline Engagement continuo & $12.9 \%$ & $11.1 \%$ & $6.1 \%$ \\
\hline
\end{tabular}

Fonte: ns. elaborazione

Nella tavola 1 si rileva, nel triennio 2014-2016, un calo costante dei dissensi medi sulle proposte presentate dal Board, o da azionisti rilevanti in assemblea ordinaria, che si riducono dell' $8,6 \%$ tra il 2014 ed il 2015 , e dell' $8,0 \%$ nel 2016.

Pertanto trova conferma l'ipotesi 1.

Tuttavia, sia il livello di dissenso, sia il trend triennale, evidenziano profonde differenze tra i gruppi di emittenti analizzati, a seconda delle pratiche di engagement adottate. Mentre non è possibile osservare un trend definito nelle società che non effettuano alcun tipo di engagement con gli azionisti istituzionali (il dissenso medio si riduce sensibilmente, nel 2015, del 9,7\%, ma torna a crescere nel 2016); il dissenso medio negli emittenti che hanno adottato politiche di engagement risulta costantemente inferiore al resto del campione, evidenziando un calo decisamente più sensibile (-14\% nel 2015 e addirittura -45,4\% nel 2016). Un trend simile è riscontrabile nelle società che tendono ad effettuare attività di engagement in prossimità dell'assemblea, focalizzandosi su particolari proposte all'ordine del giorno. In tale categoria di emittenti, il dissenso risulta generalmente in linea con le medie del campione, risultando addirittura leggermente superiore nel 2015; al contrario, il dissenso è decisamente inferiore nei casi di engagement continuativo.

Allo scopo di affinare ulteriormente l'analisi, si è analizzato il dissenso medio delle minoranze sulle politiche di remunerazione. Il dissenso medio su tutte le proposte all'ordine del giorno può essere condizionato da ulteriori fattori, non necessariamente legati al governo societario. Ad esempio, la media risulterà necessariamente inferiore laddove la destinazione del risultato di esercizio è votata in un punto allordine del giorno separato dall'approvazione del bilancio. Tali risoluzioni, per propria
Salvatore Esposito De Falco Nicola Cucari

Sergio Carbonara co-creation. co-creation.

Un'analisi su un campione di imprese quotate 
sinergie Vol. 36, N. 106, 2018

natura, sono quelle su cui si riscontra il più basso livello di dissenso. L'impatto positivo sulla media dei voti favorevoli è ovvio, nel momento in cui tali risoluzioni sono proposte in due punti separati, aumentando il denominatore e riducendo il numeratore nel calcolo della media. D’altro canto, tutti gli emittenti sono tenuti a far votare le politiche di remunerazione all'assemblea annuale e, storicamente, è su tale risoluzione che si concentra il dibattito tra emittenti ed azionisti.

Tav. 2: Dissenso delle minoranze sulle politiche di remunerazione

\begin{tabular}{|l|c|c|c|}
\hline & \multicolumn{3}{|c|}{ Dissenso minoranze su politiche di remunerazione } \\
\hline & 2014 & 2015 & 2016 \\
\hline Media campione & $30.6 \%$ & $32.2 \%$ & $32.1 \%$ \\
\hline No engagement & $38.1 \%$ & $33.6 \%$ & $36.2 \%$ \\
\hline Solo in prossimità di Assemblee & $23.1 \%$ & $41.1 \%$ & $39.9 \%$ \\
\hline Engagement continuo & $22.4 \%$ & $19.1 \%$ & $14.8 \%$ \\
\hline
\end{tabular}

Fonte: ns. elaborazione

Lanalisi del dissenso medio sulle politiche di remunerazione (cfr. Tav. 2) sembrerebbe confermare l'ipotesi per cui l'engagement può generare co-creazione - intesa come clima ideale di confronto tra proprietà e management nella definizione delle migliori pratiche di governance - solo se realizzato in maniera continuativa e non finalizzata esclusivamente a rispondere a situazioni contingenti di difficoltà (elevati dissensi su politiche di remunerazione o necessità di elevati consensi per approvare specifiche risoluzioni).

L'ipotesi 2 è confermata in primo luogo dal trend decisamente discendente dei dissensi (-14,7\% nel 2015 e -22,5\% nel 2016), peraltro sempre mediamente inferiori rispetto al resto del campione, nelle società che adottano politiche di engagement continuo. Una seconda conferma arriva anche dall'analisi delle società che attivano l'engagement solo in prossimità di assemblee: l'impennata dei dissensi tra il 2014 ed il 2015 $(+77,9 \%)$ sembra evidenziare come tale tipologia di engagement miri esclusivamente a reagire ad una situazione di difficoltà, senza ottenere benefici significativi per l'emittente o gli azionisti (come conferma la sostanziale stabilità dei dissensi medi nel 2016, in calo solo del 2,9\%, contro un $-22,5 \%$ delle società con politiche di engagement continuo).

\section{Conclusioni}

Inserendosi nel più ampio filone di studi sullo shareholder activism, il presente lavoro rappresenta una prima verifica, nel contesto italiano, degli aspetti peculiari dello shareholder engagement, in unottica di co-creazione.

Va osservato che non sempre la presenza di un dialogo attivo tra tutti gli attori in gioco determina condizioni di governo ottimali tra maggioranza e minoranza. In alcuni casi il grado di consonanza tra gli azionisti (quest'ultima intesa come convergenza degli interessi) può generare effetti diversi sugli equilibri di governo. 
Le condizioni per la co-creazione di valore tra gli attori in gioco possono, infatti, dipendere dall'interazione tra dialogo e consonanza tra gli shareholders. In particolare, il livello di dialogo è frutto di unazione di shareholder engagement; il livello di consonanza, invece, si riferisce al grado di convergenza degli interessi tra gli azionisti.

Fig. 1: Condizioni per la co-creazione di valore tra gli shareholders

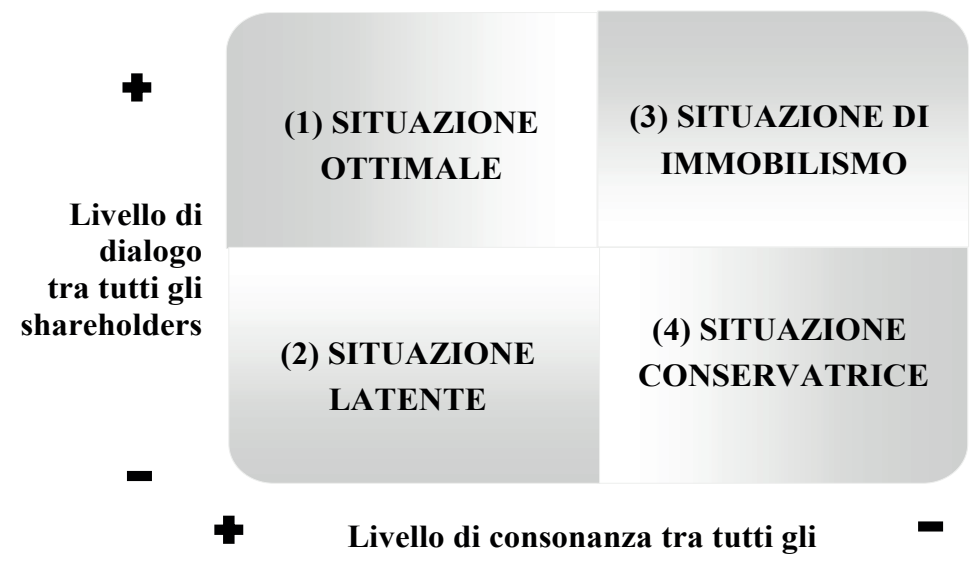

Fonte: ns. elaborazione

Si delineano, pertanto, 4 situazioni tipo, che meritano un ulteriore approfondimento in studi futuri (Cfr. Fig. 1):

1. Situazione ottimale (quadrante 1): in cui l'azione di shareholder engagement induce un dialogo ed una discussione aperta, condivisa e svolta in modo continuo tra tutti gli azionisti. In tale situazione l'efficacia dell'engagement è amplificata dalla convergenza degli interessi tra gli azionisti; quest'ultima resa evidente da un basso dissenso delle minoranze verso le decisioni assembleari. Nella situazione definita ottimale, quindi, si creano le condizioni ideali per cui l'engagement favorisce l'insorgere di un processo di co-creation tra tutti gli attori in gioco;

2. Situazione latente (quadrante 2): nei casi in cui vi è un basso livello di dialogo, accompagnato da un elevata affinità di interessi, le condizioni per la co-creazione di valore possono raggiungersi attraverso l'intensificazione delle azioni di engagement eterodirette da soggetti esterni (quali i proxy solicitor), ai quali è demandato il compito di facilitare il flusso di informazioni dagli emittenti agli investitori istituzionali, contribuendo a migliorare la comprensione delle proposte avanzate. In tali casi l'engagement può ridurre l'elevato livello di dissenso dovuto all'assenza di dialogo e, quindi, alla persistenza di condizioni di asimmetria informativa. Al pari la presenza di interessi condivisi potrebbe rappresentare un terreno fertile per le azioni di engagement;

3. Situazione di immobilismo (quadrante 3): nei casi di elevato livello di dialogo accompagnato da una divergenza di interessi tra 
sinergie

Vol. 36, N. 106, 2018

maggioranza e minoranza, si possono determinare situazioni di immobilismo decisionale, tanto più spinto se posto in relazione al grado di concentrazione proprietaria: più è alto il peso dell'azionista di maggioranza, più è marcato l'immobilismo. Agli shareholders, sebbene parzialmente coinvolti prima delle assemblee, non viene assicurata una concreta risposta alle loro richieste e aspettative. In tale situazione, l'azione di shareholder engagement non è in grado di creare le condizioni per la co-creazione di valore;

4. Situazione conservatrice (quadrante 4): è una situazione in cui lo shareholder engagement non ha ancora trovato applicazione in quanto, da un lato, l'azionista di controllo non avverte la necessità di confrontarsi con altri investitori e, dall'altro lato, non si creano le condizioni per un clima di co-creazione. In tale contesto il livello di dissenso è alto per cui, a meno che non si verifichi una situazione estrema (ad esempio una OPA ostile), l'azionista di maggioranza può continuare ad estrarre elevati livelli di benefici privati di controllo.

In conclusione, nel lavoro si è voluto evidenziare come lo shareholder engagement può dare luogo a forme di co-creazione di valore in unottica win-win (Spohrer et al., 2008), qualora da risorsa potenziale si trasformi in un effettivo beneficio specifico per tutti gli azionisti (Polese et al., 2015). In quest'ottica si inserisce il recente dibattito circa una maggiore collaborazione tra imprese, azionisti e altri stakeholder con il fine di ottenere un valore a lungo termine (Lipton, 2016). Ciò è quanto sostiene anche l'International Business Council of the World Economic Forum, che ritiene necessario riconoscere una nuova tendenza della corporate governance, con un riequilibrio dei poteri decisionali tra azionisti di controllo e azionisti di minoranza. In altre parole si vuole attribuire, alla corporate governance, il ruolo di allineare gli interessi degli attori in gioco, così da facilitare una più semplice convergenza dei processi decisionali.

La ricerca condotta non è naturalmente priva di limiti. In primo luogo, il sample di riferimento e il periodo temporale di analisi. In secondo luogo, la costruzione e la misurazione della value co-creation, che interpreta l'engagement come azione di partecipazione al dialogo. L'engagement e l'interazione, negli studi di marketing, sono riconosciuti come elementi importanti della value co-creation (Bendapudi e Leone 2003; Ranjan e Read, 2016). In quest'ottica, l'applicazione degli elementi della value cocreation, tipici di un contesto di marketing, ad un ambito di governance aziendale, necessitano di ulteriori sviluppi empirici.

Malgrado questi limiti, l'analisi empirica fornisce spunti per sviluppi futuri, alimentando il dibattito sull'importanza dello shareholder engagement. In particolare, si sostiene che lo shareholder engagement possa divenire parte fondamentale del "buon governo" societario, e che lo stesso possa indurre ad un ripensamento del ruolo dell'assemblea, luogo dove è maggiormente osservabile sia lo shareholder activism, sia lo shareholder engagement. In effetti, è proprio lo shareholder engagement che permette la realizzazione di una governance attenta ad un'efficiente interazione degli interessi di tutti gli stakeholders, favorendo, in tal modo, un cambiamento di prospettiva da una governance interna ad una esterna (Esposito De Falco, 2010). Se infatti l'attenzione alla governance "interna" è più legata 
alla capacità di monitoraggio del Board, nonché alla composizione e funzionamento dello stesso, la governance "esterna" mira ad un diverso rapporto con gli azionisti, ad una rivalutazione delle forme di engagement e, pertanto, ad una diversa dialettica societaria.

Un ulteriore spunto per future ricerche potrebbe derivare dal confronto con altri contesti e tipologie di engagement. Infine, tra le principali implicazioni manageriali derivanti dallo studio, si segnala la possibilità, per gli emittenti che non hanno ancora attuato politiche continuative di shareholder engagement, di disporre di dati che dimostrano l'effetto dello stesso in termini di minore dissenso su specifiche proposte.

\section{Bibliografia}

ACHARYA V.V., VOLPIN P.F. (2010), "Corporate governance Externalities", Review of Finance, vol. 14, n. 1, pp. 1-33.

AGUILERA R.V., DESENDER K., BEDNAR M.K., LEE J.H. (2015), "Connecting the dots: Bringing external corporate governance into the corporate governance puzzle", Academy of Management Annals, vol. 9, n. 1, pp. 483573.

AGUILERA R., JUDGE W., TERJESEN S. (2016), "Corporate governance Deviance", Academy of Management Review, pubblicato on line prima della stampa il 18 luglio 2016.

AMEER R., RAHMAN R.A. (2009), “The impact of minority shareholder watchdog group activism on the performance of targeted firms in Malaysia", Asian Academy of Management Journal of Accounting and Finance, vol. 5, n. 1, pp. 67-92.

ASSONIME (2015), La Corporate governance in Italia: autodisciplina, remunerazioni e comply-or-explain, Roma.

BARILE S., GATTI M. (2007), "Corporate governance e creazione di valore nella prospettiva sistemico-vitale”, Sinergie, n. 73-74, pp. 151-168.

BENDAPUDI N., LEONE R.P. (2003), "Psychological implications of customer participation in co-production", Journal of Marketing, vol. 67, n. 1, pp. 14-28.

CHAPMAN AND CUTLER LLP (a cura di) (2016), Considering a Shareholder Engagement Policy. The What, Why and How, New York, Available on: http://www.chapman. com/insights-publications-Shareholder_ Engagement_Policy.html

CHUAH K. (2017), "Carrot or Stick? Strategic Corporate governance and the Incentivization of Attention to Environmental, Social, and Governance Issues", Available at SSRN: https://ssrn.com/abstract=3090302

CHUNG H.H., WYNN J.P (2014), "Corporate governance, directors' and officers' insurance premiums and audit fees", Managerial Auditing Journal, vol. 29, pp. 173-195.

CHUNG H., TALAULICAR T. (2010), "Forms and effects of shareholder activism", Corporate governance: An International Review, vol. 18, n. 4, pp. 253-257.

COLLOVÀ C., PRESTIDGE S. (2015), Corporate governance: long-term shareholder engagement, European Parliamentary Research Service.

CONFERENCE Board Governance CENTER (2016), Guidelines for Investor Engagement, Research Report, USA.
Salvatore Esposito De Falco Nicola Cucari

Sergio Carbonara

Shareholder engagement co-creation.

Un'analisi su un campione di imprese quotate 
sinergie Vol. 36, N. 106, 2018

CONSOB (2016), Relazione per l'anno 2015, Roma.

CRANE A., PALAZZO G., SPENCE L.J., MATTEN D. (2014), "Contesting the value of 'Creating Shared value", California Management Review, vol. 56, n. 2, pp. 130-151.

DAVIS J.H., SCHOORMAN F.D., DONALDSON L. (1997), "Toward a Stewardship Theory of management”, Academy of Management review, vol. 22, n. 1, pp. 20-47.

DEMBEK K., SINGH P., BHAKOO V. (2016), "Literature Review of Shared value: A Theoretical Concept or a Management Buzzword?”, Journal of Business Ethics, vol. 137, n. 2, pp. 231-267.

DOIDGE C., KAROLYI A., LINS V., MILLER P., STULZ M. (2009), "Private Benefits of Control, Ownership, and the Cross-Listing Decision”, Journal of Finance, vol. 64, n. 1, pp. 425-466.

DONALDSON T., PRESTON L.E. (1995), "The stakeholder theory of the corporation: Concepts, evidence, and implications", Academy of Management Review, vol. 20, n. 1, pp. 65-91.

DYCK A., ZINGALES L. (2004), "Private benefits of control: An international comparison", The Journal of Finance, vol. 59, n. 2, pp. 537-600.

EHRHARDT O., NOWAK E. (2003), "Private benefits and minority shareholder expropriation (or what exactly are private benefits of control?", in EFA 2003 Annual Conference Paper, n. 809.

ERTIMUR Y., FERRI F., MUSLU V. (2010), "Shareholder Activism and CEO Pay", Review of Financial Studies, vol. 24, n. 2, pp. 535-592.

ESPOSITO DE FALCO S. (2006) (a cura di), Evoluzione e prospettive negli studi sulla governance tra economia e diritto societario, Edizioni Scientifiche Italiane, Napoli.

ESPOSITO DE FALCO S. (2010), "La Corporate governance negli studi sul governo d'impresa. Approcci prevalenti e strumenti di controllo", in Pellicano M. e Ciasullo M.V. (a cura di), La visione strategica dell'impresa, Giappichelli Editori, Torino.

ESPOSITO DE FALCO S. (2014), La corporate governance per il governo dell'impresa, McGraw-Hill, Milano.

ESPOSITO DE FALCO S. (2017), I rapporti di potere nel sistema proprietario. Il difficile equilibrio tra maggioranza e minoranza, Cedam, Padova.

ESPOSITO DE FALCO S., CUCARI N., SORRENTINO E. (2016), "Voting dissent and corporate governance structures: the role of Say on pay in a comparative analysis", Corporate Ownership and Control, vol. 13, n. 4, pp. 188-197.

FERRARO, F., BEUNZA D. (2014), "Why Talk? A Process Model of Dialogue in Shareholder Engagement" SSRN Scholarly Paper n. ID 2419571, Rochester, NY: Social Science Research Network. http://papers.ssrn.com/ abstract $=2419571$

FREEMAN R.E. (1994), "The politics of stakeholder theory: Some future directions", Business Ethics Quarterly, vol. 4, n. 4, pp. 409-421.

GEORGESON (2011), Annual Corporate governance Review, Report available on: http://www.computershare-na.com/sharedweb/georgeson/acgr/acgr2011. pdf

GIFFORD J. (2012), "Effective shareholder engagement: The factors that contribute to shareholder salience", in Hebb T. (a cura di), The next generation of responsible investing, vol. 1, Springer Science, London, pp. 83-106. 
GOLDSTEIN M. (2014), Defining engagement: An update on the evolving relationship between shareholders, directors and executives, edizione a cura di Institutional Shareholder Services for the Investor Responsibility Research Center Institute, New York, Available on: https://irrcinstitute. org/wp-content/uploads/2015/09/engagement-between-corporationsand-investors-at-all-time-high1.pdf

GOLINELLI G.M. (2000), L'approccio sistemico al governo dellimpresa. L'impresa sistema vitale, vol. 1, Cedam, Padova.

GOODMAN J., LOUCHE C., VAN CRANENBURGH K.C., ARENAS D. (2014), "Social shareholder engagement: The dynamics of voice and exit", Journal of Business Ethics, vol. 125, n. 2, pp. 193-210.

GORANOVA M., RYAN L.V. (2014), "Shareholder activism a multidisciplinary review”, Journal of Management, vol. 40, n. 5, pp. 1230-1268.

HIRSCHMAN A.O. (1970), Exit, voice, and loyalty: Responses to decline in firms, organizations, and states, Harvard University Press.

HOCKERTS K., MOIR L. (2004), "Communicating corporate responsibility to investors: The changing role of the investor relations function", Journal of Business Ethics, vol. 52. n. 1, pp. 85-98.

IND N., COATES N. (2013), “The meanings of co-creation”, European Business Review, vol. 25, n. 1, pp. 86-95.

IVANOVA M.R. (2017), "Institutional investors as stewards of the corporation: Exploring the challenges to the monitoring hypothesis", Business Ethics: A European Review, vol. 26, n. 2, pp. 175-188.

JUDGE W.Q., GAUR A., MULLER-KAHLE M.I. (2010), "Antecedents of shareholder activism in target firms: evidence from a multi-country study", Corporate governance: An International Review, vol. 18, n. 4, pp. 258-273.

KLEIN A., ZUR E. (2009), "Entrepreneurial shareholder activism: Hedge funds and other private investors", The Journal of Finance, vol. 64, n. 1, pp. 187-229.

KORHONEN H. (2013), "Organizational needs: A co-creation and human systems perspective", Journal of Business Market Management, vol. 6, n. 4, pp. 214-22.

LA PORTA R., LOPEZ-DE-SILANES F., SHLEIFER A. (1999), “Corporate ownership around the world”, The Journal of Finance, vol. 54, n. 2, pp. 471-517.

LEE M.D.P., LOUNSBURY M. (2011), “Domesticating Radical Rant and Rage: An Exploration of the Consequences of Environmental Shareholder Resolutions on Corporate Environmental Performance", Business and Society, vol. 50, n. 1, pp. 155-188.

LETZA S., SUN X., KIRKBRIDE J. (2004), "Shareholding versus stakeholding: A critical review of corporate governance", Corporate governance: An International Review, vol. 12, n. 3, pp. 242-262.

LIPTON M. (2016), The New Paradigm for Corporate governance. Harvard Law School Forum on Corporate governance and Financial Regulation, February 2016, Available on: https://corpgov.law.harvard.edu/2016/02/03/the-newparadigm-for-corporate-governance

LOVELOCK C., GUMMESSON E. (2004), "Whither services marketing? In search of a new paradigm and fresh perspectives", Journal of Service Research, vol. 7, n. 1, pp. 20-41.

LYDENBERG S. (2007), "Universal investors and socially responsible investors: A tale of emerging affinities", Corporate governance: An International Review, vol. 15, n. 3, pp. 467-477.
Salvatore Esposito De Falco Nicola Cucari

Sergio Carbonara

Shareholder engagement e co-creation.

Un'analisi su un campione di imprese quotate 
MALLIN C., MELIS A. (2012), "Shareholder rights, shareholder voting, and corporate performance", Journal of Management and Governance, vol. 16, n. 2, pp. 171-176.

MCNULTY T., ZATTONI A., DOUGLAS T. (2013), "Developing corporate governance research through qualitative methods: A review of previous studies", Corporate governance: An International Review, vol. 21, n. 2, pp. 183-198.

MEHTA T. (2013), Shareholder Engagement: Maximizing the Shareholder Relationship, Executive Compensation Insights, Available on:

https://www.issgovernance.com/file/publications/ MaximizingTheShareholderRelationshipvol._13.3.pdf

MITCHELL R.K., AGLE B.R., WOOD D.J. (1997), "Toward a Theory of Stakeholder Identification and Salience: Defining the Principle of Who and What Really Counts", Academy of Management Review, vol. 22, n. 4, pp. 853-886.

NORMANN R., RAMIREZ R. (1994), Designing Interactive Strategy. From Value Chain to Value Costellation, John Wiley \& Sons, Chicester, England.

OTHMAN S., BORGES W.G. (2015), "Shareholder Activism in Malaysia: Is it Effective?”, Procedia-Social and Behavioral Sciences, vol. 172, pp. 427-434.

POLESEF., IANDOLOF., CARRUBO L. (2015), “Co-creare valore compartecipando valori. Un contributo in ottica service, tra fruizione e compartecipazione", Atti del XXVII Convegno annuale di Sinergie - Heritage, management e impresa: quali sinergie?, Termoli, 9-10 Luglio.

PORTER M.E., KRAMER M.R. (2006), "Strategy and society: The link between competitive advantage and corporate social responsibility", Harvard Business Review, vol. 84, n. 12, pp. 78-92.

PORTER M.E., KRAMER M.R. (2011), “Creating shared value”, Harvard Business Review, vol. 89, n. 1/2, pp. 62-77.

PRAHALAD C.K., RAMASWAMY V. (2004), "Co-creation experiences: The next practice in value creation", Journal of Interactive Marketing, vol. 18, n. 3, pp. 5-14.

RANJAN K.R., READ S. (2016), "Value co-creation: concept and measurement", Journal of the Academy of Marketing Science, vol. 44, n. 3, pp. 290-315.

REHBEIN K., LOGSDON J.M., VAN BUREN H.J. (2013), “Corporate responses to shareholder activists: Considering the dialogue alternative", Journal of Business Ethics, vol. 112, n. 1, pp. 137-154.

RENNEBOOG L., SZILAGYI P. (2013), "Shareholder engagement at European general meetings", in Belcredi M., Ferrarini G., Boards and shareholders in European listed companies. Facts, contexts and reform proposals, Cambridge University Press, Cambridge, pp. 251-315.

RÖNDELL J.G., SÖRHAMMAR D., GIDHAGEN M. (2016), “Co-governance in the consumer engagement process: facilitating multi-beneficial value creation", Journal of Strategic Marketing, vol. 24, n. 3-4, pp. 327-345.

SPOHRER J., ANDERSON L., PASS N., AGER T. (2008), Service Science e Service Dominant Logic, Otago Forum 2, pp. 4-18.

STRAMPELLI G. (2017), "Knocking at the Boardroom Door: A Transatlantic Overview of Director-Institutional Investor Engagement in Law and Practice", Virginia Law and Business Review, Forthcoming. Available at SSRN: https://ssrn.com/abstract=3044278 
VARGO S.L., LUSCH R.F. (2004), "Evolving to a new dominant logic for marketing”, Journal of marketing, vol. 68, n. 1, pp. 1-17.

VON KROGH G., ROSSI-LAMASTRA C., HAEFLIGER S. (2012), "Phenomenonbased research in management and organization science: When is it rigorous and does it matter?", Long Range Planning, vol. 45, n. 4, pp. 277298.

YOUNG M.N., PENG M.W., AHLSTROM D., BRUTON G.D., JIANG Y. (2008), "Corporate governance in emerging economies: A review of the principalprincipal perspective", Journal of Management Studies, vol. 45, n. 1, pp. 196220.

\section{Academic or professional position and contacts}

Salvatore Esposito De Falco

Full Professor of Management

Sapienza University of Roma - Italy

e-mail: salvatore.espositodefalco@uniroma1.it

\section{Nicola Cucari}

Research Assistant

University of Salerno - Italy

e-mail: ncucari@unisa.it

\section{Sergio Carbonara}

Founder of Frontis Governance, Partner ECGS, Roma

e-mail: s.carbonara@frontisgovernance.com
Salvatore Esposito De Falco Nicola Cucari

Sergio Carbonara

Shareholder engagement e co-creation.

Un'analisi su un campione di imprese quotate 